\title{
PURELY INSEPARABLE POINTS ON CURVES OF HIGHER GENUS
}

\author{
MinHYONG KIM
}

1.

Let $k$ be a field of characteristic $p>0$ and let $K$ be a function field of one variable over $k$, i.e., $K \simeq k(t)[s] /(h(t, s))$ where $h \in k[t, s]$ is a polynomial irreducible in $\bar{k}[t, s]$. Now let $f(x, y)$ be a polynomial with coefficients in $K$. We are interested in $K$-rational solutions to the equation

$$
f(x, y)=0 \text {. }
$$

For example, the equation

$$
x^{l}-y^{l}=t^{l}-1
$$

has solution $(t, 1)$ in $K=k(t)$.

Given any such $f$ we can keep 'twisting' its coefficients with the Frobenius map of $K$ and get new polynomials $f^{(n)}(x, y)$ (by which we denote the $n$th twist) and new equations $f^{(n)}(x, y)=0$.

The question we wish to ask is whether $f^{(n)}=0$ can keep having new solutions as we increase $n$. An 'old' solutions for the twisted equation means the following: If $(x, y)$ is a solution for $f=0$, then obviously, $(x, y)^{(n)}:=\left(x^{p^{n}}, y^{p^{n}}\right)$ is a solution for $f^{(n)}=0$; in the example above, the equation $f^{(1)}=0$ is

$$
x^{l}-y^{l}=t^{l p}-1
$$

and we have the twisted solution $\left(t^{p}, 1\right)$.

By new solutions to $f^{(n)}=0$ then, we mean solutions which don't arise by twisting solutions to $f^{(n-1)}=0$.

One way in which infinitely many $f^{(n)}=0$ can have new solutions is if $f$ has coefficients in some finite field ( $f$ is a 'constant' polynomial). For then, infinitely many $f^{(n)}$ will be equal to $f$, and hence, have solutions which are not Frobenius twists (if $f$ does).

For example, if $K=k(t)[s] /\left(s^{l}-t^{l}+1\right)$ ( with $l$ prime to $p$ ), then the equation $x^{l}-y^{l}=1$ has the $K$-rational solution $(t, s)$, and all its twists have the new solution $(t, s)$. Obviously, such a thing can happen also with an $f$ which admits a $K$-rational change of variables to such a constant polynomial. The purpose of this note is to show that this is essentially all that can happen for higher genus equations, that is:

Received May 15, 1997. 
Theorem 1. Suppose $f(x, y)=0$ defines a curve over $K$ whose genus over $\bar{K}$ is at least 2 and suppose infinitely many of the equations $f^{(n)}(x, y)=0$ have new solutions. Then there is a change of variables in some finite extension of $K$ (including a possible extension of $k$ ) which sends $f$ to a polynomial with coefficients in a finite field.

More precisely, the 'change of variables' here refers to a birational map of curves.

By combining this with standard finiteness for each individual curve $f^{(n)}=0$ (geometric Mordell conjecture), we get the following

Corollary 1. Suppose $f(x, y)=0$ defines a curve over $K$ whose genus over $\bar{K}$ is at least 2 and suppose it is not birational over any finite extension to a curve defined over a finite field. Then the set of its solutions in the field $K^{1 / p^{\infty}}:=\lim _{n} K^{1 / p^{n}}$ is finite.

For the theorem, our two examples above illustrate such a change of variables, and why an extension may be necessary. The field $K$ in this case is $k(t)$ and the extension is $k(t)[s] /\left(s^{l}-t^{l}+1\right)$ while the change of variables is $(x, y) \mapsto(x / s, y / s)$. The existence of such a 'constant form' for the equation over some extension may cause infinitely many new solutions as follows: Let $l$ be a prime different from $p$ and let $n$ range over integer multiples of $l-1$. Then we see that $p^{n}-1=m l$ for some $m$, and hence,

$$
\left[\left(t^{l}-1\right)^{m} t\right]^{l}-\left[\left(t^{l}-1\right)^{m}\right]^{l}=\left(t^{l}-1\right)^{p^{n}-1} t^{l}-\left(t^{l}-1\right)^{p^{n}-1}=t^{l p^{n}}-1,
$$

so that $\left(\left(t^{l}-1\right)^{m} t,\left(t^{l}-1\right)^{m}\right)$ are new solutions to $f^{(n)}=0$. One checks easily that the constant form is responsible for these solutions.

\section{2.}

Proof of Theorem:

It suffices to prove the theorem after a finite extension of $K$ (which may include a finite extension of $k$ ), so we may assume that there is a projective smooth model for $f(x, y)=0$ which has semistable reduction. That is, the curve $f(x, y)=0$ over $K$ is birational to the generic fiber of a map $\pi: X \rightarrow B$ where $X$ is a projective smooth surface and $B$ a projective smooth curve over $k$, such that

i) $\pi$ has smooth generic fiber of genus at least two,

ii) all of its geometric fibers are reduced with at worst normal crossing singularities;

iii) and its fibers contain no $(-1)$-curves. 
Then a new solution to $f^{(n)}=0$ will give rise to a diagram

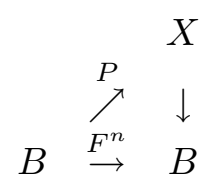

with the property that the map $P^{*} \Omega_{X} \rightarrow \Omega_{B}$ is non-zero.

Most of the proof is contained in the following

Claim: Suppose the fibration $\pi: X \rightarrow B$ is not isotrivial. Then a diagram as above exists for at most finitely many $n$.

Proof. Assume that we have a separable map $P: B \rightarrow X$ such that $\pi \circ P=F^{n}$ for $n \geq 1$. Let $S \subset B$ be the set of singular values of the fibration and let $D \subset X$ be the union of the singular fibers, that is, $D=\pi^{-1}(S)$. Let $\Omega(\log D)$ be the sheaf of differentials with log poles along the normal crossing divisor $D$. This fits into an exact sequence

$$
0 \rightarrow \pi^{*} \Omega_{B}(S) \rightarrow \Omega(\log D) \rightarrow \omega \rightarrow 0
$$

since the dualizing sheaf is isomorphic to the sheaf of relative log differentials. To see this, note that both are invertible sheaves and $c_{1}(\Omega(\log D))=K_{X}+D$ so that $c_{1}(\Omega(\log D))-c_{1}\left(\pi^{*} \Omega_{B}(S)\right)=K_{X}-\pi^{*} K_{B}$.

We have a non-zero map $P^{*} \Omega(\log D) \rightarrow \Omega_{B}\left(\left(F^{n}\right)^{-1} S\right)=\Omega_{B}(S)$ which factors to $P^{*} \omega \rightarrow \Omega_{B}(S)$, by the inseparability. Thus, $\operatorname{deg} P^{*} \omega \leq 2 g_{B}-2+s$, where $s=|S|$.

Now, since $\omega$ is a big line bundle $[\mathrm{Sz}]$, this degree bound implies that the divisor $P_{*}(B)$ lies inside a bounded algebraic family. In particular, only finitely many degrees for the intersection with a fiber is possible. This implies that in $\pi \circ P=F^{n}, n$ is bounded.

Suppose $f^{(n)}=0$ has new solutions for infinitely many $n$. By the claim above this implies that $X / B$ is isotrivial. So replacing $B$ by a base change, we can assume that $X$ is birational to $B \times C$, where $C$ is a smooth projective curve over $k$ of genus at least 2 . Then a new solution of degree $p^{n}$ corresponds to a (nonconstant) separable map $g: B \rightarrow\left(F^{n}\right)^{*} C$, where the Frobenius in this case is the Frobenius of $k$. This must exist for infinitely many $n$. By a theorem of Severi $[S]$, there are only finitely many function fields of genus at least two separably contained in the function field of $B$. That is, infinitely many of the $\left(F^{n}\right)^{*} C$ are isomorphic. This implies that some Frobenius pull-back of $C$ is defined over a finite field (by considering the corresponding moduli points). 


\section{Acknowledgement}

I am grateful to Felipe Voloch for discussions related to this paper.

\section{References}

[S] P. Samuel, Compléments á un article de Hans Grauert sur la conjecture de Mordell, Publ. Math. I.H.E.S. (1965), 311-318.

[Sz] L. Szpiro, 'Propriétés numériques du faisceaux dualisant relatif,' in 'Pinceaux de courbes de genre au moins deux', Astérisque 86 (1981).

Department of Mathematics, University of Arizona, Tucson, AZ 85721

E-mail address: kim@math.arizona.edu 